\title{
Effects of Suction-Injection-Combination (SIC) on the onset of Rayleigh-Bénard Electroconvection in a Micropolar Fluid
}

\author{
S Pranesh*, Sameena Tarannum ${ }^{\dagger}$ and Riya Baby $\ddagger$
}

\begin{abstract}
The effect of Suction - injection combination on the onset of Rayleigh - Bénard electroconvection micropolar fluid is investigated by making a linear stability analysis. The Rayleigh-Ritz technique is used to obtain the eigenvalues for different velocity and temperature boundary combinations. The influence of various parameters on the onset of convection has been analysed. It is found that the effect of Prandtl number on the stability of the system is dependent on the SIC being pro-gravity or anti-gravity. A similar Pe-sensitivity is found in respect of the critical wave number. It is observed that the fluid layer with suspended particles heated from below is more stable compared to the classical fluid layer without suspended particles.
\end{abstract}

Keywords: Micropolar fluids, Rayleigh-Bénard convection, Suction-injection-combination and electroconvection.

\footnotetext{
* Department of Mathematics, Christ University, Bangalore, India; pranesh.s@christuniversity.in

† Department of Professional Studies, Christ University, Bangalore, India; sameena.tarannum@christuniversity.in

‡ Research Scholar, Department of Mathematics, Christ University, Bangalore, India; riyababy.p@gmail.com
}

Received: April 2015. Reviewed: June 2015 


\section{Introduction}

Convection in Eringen's micropolar fluid has been the subject of intensive study because of the remarkable physical properties of the fluid as well as its practical applications (see Power [1], Lukasazewicz [2] and Eringen [3]). The Rayleigh-Bénard convection in Eringen's micropolar fluid [4] have been studied by many of authors (Datta and Sastry [5], Ahmadi [6], Bhattacharya and Jena [7], Siddheshwar and Pranesh [8-11], Pranesh and Riya Baby [12] and Pranesh and Arun Kumar [13]).

The classical Rayleigh problem of onset of convection instabilities in a horizontal layer of fluid heated from below has its origin in the experimental observations of Bénard [14]. In the standard classical problem there is no flow across the horizontal boundaries. A modified problem, where the boundaries are permeable and there is injection of fluid at one boundary and suction of fluid at the other boundary, was studied by Sharvartsblat [15]. He pointed out, that the problem is of interest because of possibilities of controlling the convection instability by adjusting of SIC. The effect of SIC is in general quit complex because not only the basic temperature profile is altered, but in the perturbation equations contribution arises from the convection of both temperature and velocity, and there is an interaction between Prandtl number comes into play. Nield [16] considered the classical problem for viscous fluid and studied analytically the effect of SIC on the onset of convection in a fluid layer between permeable horizontal boundaries. He gave a new theory for the destabilizing effects of SIC, when flow is from a dynamically rigid and thermally conducting boundary to a dynamically free and thermally insulating boundary. He also pointed that when Prandtl number is close to unity the amount of destabilization is small but this is not true when Prandtl number is either large or small. Many authors (Siddheshwar and Pranesh [17], Shivakumara and Suma [18], Murty and Ramana Rao [19] and Murty $[20,21])$ have studied the effect of SIC on Newtonian and non-Newtonian fluid using classical Fourier law.

The objective of this paper is to study the effect of suctioninjection-combination on the onset of Rayleigh-Bénard electroconvection in a micropolar fluid. 


\section{Mathematical Formulation}

Consider an infinite horizontal layer of a Boussinesquian, electrically conducting micropolar fluid of depth ' $\mathrm{d}$ ' permeated by an externally applied uniform magnetic field normal to the layer. A cartesian coordinate system is taken with origin in the lower boundary and z-axis vertically upwards. Let $\Delta \mathrm{T}$ be the temperature difference between the upper and lower boundaries (see Figure (1)). A constant flow in the vertical direction, known as SIC, is maintained at the boundaries.

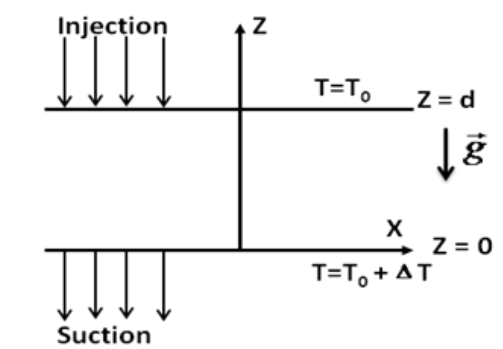

$$
\text { Pro - Gravity }\left(\mathbf{w}_{0}<0\right)
$$

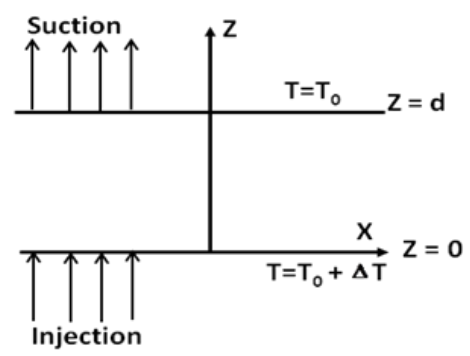

Anti - Gravity $\left(w_{0}>0\right)$

Fig 1: Schematic diagram of the flow configuration.

The governing equations for the Rayleigh-Bénard situation in a Boussinesquain micropolar fluid are:

Continuity equation:

$\nabla \cdot \vec{q}=0$,

Conservation of linear momentum:

$\rho_{0}\left[\frac{\partial \overrightarrow{\mathrm{q}}}{\partial \mathrm{t}}+(\overrightarrow{\mathrm{q}} \cdot \nabla) \overrightarrow{\mathrm{q}}\right]=-\nabla \mathrm{p}-\rho \overrightarrow{\mathrm{g}} \widehat{\mathrm{k}}+(2 \zeta+\eta) \nabla^{2} \overrightarrow{\mathrm{q}}+\zeta \nabla \times \vec{\omega}+(\overrightarrow{\mathrm{P}} \cdot \nabla) \overrightarrow{\mathrm{E}}$,

Conservation of angular momentum:

$\rho_{\mathrm{o}} \mathrm{I}\left[\frac{\partial \vec{\omega}}{\partial \mathrm{t}}+(\overrightarrow{\mathrm{q}} \cdot \nabla) \vec{\omega}\right]=\left(\lambda^{\prime}+\eta^{\prime}\right) \nabla(\nabla \cdot \vec{\omega})+\left(\eta^{\prime} \nabla^{2} \vec{\omega}\right)+\zeta(\nabla \times \overrightarrow{\mathrm{q}}-2 \vec{\omega})$,

Conservation of energy:

$\frac{\partial \mathrm{T}}{\partial \mathrm{t}}+(\overrightarrow{\mathrm{q}} . \nabla) \mathrm{T}=\frac{\beta}{\rho \mathrm{C}_{\mathrm{v}}}(\nabla \times \vec{\omega}) \cdot \nabla \mathrm{T}+\chi \nabla^{2} \mathrm{~T}$, 
Equation of state:

$\rho=\rho_{\mathrm{o}}\left[1-\alpha\left(\mathrm{T}-\mathrm{T}_{\mathrm{O}}\right)\right]$,

Equation of state for dielectric constant:

$\varepsilon_{\mathrm{r}}=\left(1+\chi_{\mathrm{e}}\right)-\mathrm{e}\left(\mathrm{T}-\mathrm{T}_{0}\right)$,

Faraday's law:

$$
\nabla \times \overrightarrow{\mathrm{E}}=0, \overrightarrow{\mathrm{E}}=\nabla \phi\},
$$

Equation of polarisation field:

$$
\left.\nabla .\left(\varepsilon_{0} \overrightarrow{\mathrm{E}}+\overrightarrow{\mathrm{P}}\right)=0, \overrightarrow{\mathrm{p}}=\varepsilon_{0}\left(\varepsilon_{\mathrm{r}}-1\right) \overrightarrow{\mathrm{E}}\right\} .
$$

where, $\vec{q}$ is the velocity, $\rho_{0}$ is density of the fluid at temperature $T$ $=\mathrm{T}_{0}, \mathrm{p}$ is the pressure, $\rho$ is the density, $\overrightarrow{\mathrm{g}}$ is acceleration due to gravity, $\zeta$ is coupling viscosity coefficient or vortex viscosity, $\overrightarrow{\mathrm{P}}$ is dielectric polarization, $\overrightarrow{\mathrm{E}}$ is the electric field, $\lambda$ and $\eta$ are the bulk and shear spin-viscosity coefficients, $\vec{\omega}$ is the angular velocity, I is moment of inertia, $\lambda^{\prime}$ and $\eta^{\prime}$ are bulk and shear spin-viscosity coefficients, $\mathrm{T}$ is the temperature, $\chi$ is the thermal conductivity, $\beta$ is micropolar heat conduction coefficient, $\alpha$ is coefficient of thermal expansion, determining how fast the density decreases with temperature, $\sigma$ is electrical conductivity, $\varepsilon_{\mathrm{r}}$ is the dielectric constant, $\chi_{\mathrm{e}}$ is electric susceptibility, $\varepsilon_{\mathrm{o}}$ is the electric permittivity of free space and $\phi$ is the electric scalar potential.

\section{Basic State}

The basic state of the fluid being quiescent is described by

$$
\left.\begin{array}{l}
\overrightarrow{\mathrm{q}}_{\mathrm{b}}=\mathrm{w}_{0} \hat{\mathrm{k}}, \vec{\omega}_{\mathrm{b}}=0, \mathrm{p}=\mathrm{p}_{\mathrm{b}}(\mathrm{z}), \rho=\rho_{\mathrm{b}}(\mathrm{z}), \overrightarrow{\mathrm{E}}=\overrightarrow{\mathrm{E}}_{\mathrm{b}}(\mathrm{z}), \\
\overrightarrow{\mathrm{P}}=\overrightarrow{\mathrm{P}}_{\mathrm{b}}(\mathrm{z}), \mathrm{T}=\mathrm{T}_{\mathrm{b}}(\mathrm{z}), \varepsilon_{\mathrm{r}}=\varepsilon_{\mathrm{rb}}(\mathrm{z}),
\end{array}\right\} .
$$

where, $\mathrm{w}_{0}$ is the strength of the imposed constant suction (or injection) and $\hat{\mathrm{k}}$ is the unit vector in the $\mathrm{z}$-direction. The pro-gravity SIC and anti-gravity SIC is shown schematically in figure (1). 
Substituting equation (10) into basic governing equations (1)-(8), we obtain the quiescent state solutions as:

$$
\left.\begin{array}{l}
\frac{d p_{b}}{d z}=-\rho_{b} g+P_{b} \frac{\partial E_{b}}{\partial z}, \\
w_{0} \frac{d T_{b}}{d z}=\chi \frac{d^{2} T_{b}}{d z^{2}}, \\
\rho_{b}=\rho_{o}\left[1-\alpha\left(T_{b}-T_{o}\right)\right], \\
\varepsilon_{r}=\left(1+\chi_{e}\right)-e\left(T_{b}-T_{0}\right), \\
E_{b}=\left[\frac{\left(1+\chi_{e}\right) E_{0}}{\left(1+\chi_{e}\right)+\frac{e \Delta T}{h}}\right], \\
P_{b}=\varepsilon_{0} E_{0}\left(1+\chi_{e}\right)\left[1-\frac{1}{\left(1+\chi_{e}\right)+\frac{e \Delta T_{z}}{h}}\right]
\end{array}\right\},
$$

Solving equation for $T_{b}$ using the boundary conditions

$$
\begin{aligned}
& \mathrm{T}_{\mathrm{b}}=\mathrm{T}_{0}+\Delta \mathrm{T}_{\text {at }} \mathrm{z}=0, \\
& \mathrm{~T}_{\mathrm{b}}=\mathrm{T}_{0} \text { at } \mathrm{z}=\mathrm{d},
\end{aligned}
$$

we get,

$$
\mathrm{T}_{\mathrm{b}}=\mathrm{T}_{\mathrm{o}}+\frac{\Delta \mathrm{T}}{\left[1-\mathrm{e}^{\frac{\mathrm{w}_{\mathrm{o}} \mathrm{d}}{\chi}}\right]}\left[\mathrm{e}^{\frac{\mathrm{w}_{\mathrm{o}} \mathrm{z}}{\chi}-1}\right]
$$

We now superpose infinitesimal perturbations on the quiescent basic state and study the instability. 


\section{Linear Stability Analysis}

Let the basic state be disturbed by an infinitesimal thermal perturbation. We now have

$\overrightarrow{\mathrm{q}}=\mathrm{w}_{0} \hat{\mathrm{k}}+\overrightarrow{\mathrm{q}}^{\prime}, \vec{\omega}=\vec{\omega}_{\mathrm{b}}+\vec{\omega}^{\prime}, \mathrm{p}=\mathrm{p}_{\mathrm{b}}+\mathrm{p}^{\prime}, \rho=\rho_{\mathrm{b}}+\rho^{\prime}$,

$\left.\varepsilon_{\mathrm{r}}=\varepsilon_{\mathrm{rb}}+\varepsilon_{\mathrm{r}}^{\prime}, \overrightarrow{\mathrm{E}}=\overrightarrow{\mathrm{E}}_{\mathrm{b}}+\left(\mathrm{E}_{1}^{\prime} \hat{\mathrm{i}}+\mathrm{E}_{3}^{\prime} \hat{\mathrm{k}}\right), \overrightarrow{\mathrm{P}}=\overrightarrow{\mathrm{P}}_{\mathrm{b}}+\left(\mathrm{P}_{1}^{\prime} \hat{\mathrm{i}}+\mathrm{P}_{3}^{\prime} \hat{\mathrm{k}}\right),\right\}$.

$\mathrm{T}=\mathrm{T}_{\mathrm{b}}+\mathrm{T}^{\prime}$,

The primes indicate that the quantities are infinitesimal perturbations and subscript ' $b$ ' indicates basic state value.

Substituting equation (12) into equations (1)-(8) and using the basic state equations (10), we get linearised equations governing the infinitesimal perturbations in the form:

$\nabla \cdot \overrightarrow{\mathrm{q}}^{\prime}=0$

$\left.\begin{array}{rl}\rho_{\mathrm{o}} \mathrm{w}_{0}\left[\frac{\partial \overrightarrow{\mathrm{q}}^{\prime}}{\partial \mathrm{t}}\right]= & -\nabla \mathrm{p}^{\prime}-\rho^{\prime} \mathrm{g} \hat{\mathrm{k}}+(2 \zeta+\eta) \nabla^{2} \overrightarrow{\mathrm{q}}^{\prime}+\left(\zeta \nabla \times \vec{\omega}^{\prime}\right) \\ & +\left(\overrightarrow{\mathrm{P}}_{\mathrm{b}} \cdot \nabla\right) \overrightarrow{\mathrm{E}}^{\prime}+\left(\overrightarrow{\mathrm{P}}^{\prime} \cdot \nabla\right) \overrightarrow{\mathrm{E}}_{\mathrm{b}}\end{array}\right\}$,

$\rho_{\mathrm{o}} \operatorname{Iw}_{0}\left[\frac{\partial \vec{\omega}^{\prime}}{\partial \mathrm{t}}\right]=\left(\lambda^{\prime}+\eta^{\prime}\right) \nabla\left(\nabla \vec{\omega}^{\prime}\right)+\left(\eta^{\prime} \nabla^{2} \vec{\omega}^{\prime}\right)+\zeta\left(\nabla \times \overrightarrow{\mathrm{q}}^{\prime}-2 \vec{\omega}^{\prime}\right)$,

$\mathrm{w}_{0} \frac{\mathrm{dT}^{\prime}}{\mathrm{dz}}=\frac{\Delta \mathrm{T}}{\left.1-\mathrm{e}^{\frac{\mathrm{w}_{0 \mathrm{~d}}}{\chi}}\right]}\left(\frac{\mathrm{w}_{0} \mathrm{e}^{\frac{\mathrm{w}_{0} \mathrm{z}}{\chi}}}{\chi}\right)\left[\frac{\beta}{\rho_{\mathrm{o}} \mathrm{C}_{\mathrm{v}}} \nabla \times \bar{\omega}^{\prime}-\mathrm{W}^{\prime}\right]+\chi \nabla^{2} \mathrm{~T}^{\prime}$

$\rho^{\prime}=-\alpha \rho_{\mathrm{o}} \mathrm{T}^{\prime}$

$\varepsilon^{\prime}=-\varepsilon_{0} \mathrm{eT}^{\prime}$,

$\nabla .\left(\varepsilon_{0} \overrightarrow{\mathrm{E}}^{\prime}+\overrightarrow{\mathrm{P}}^{\prime}\right)=0$. 
Using equation (17) in (14), operating curl twice on the resulting equation, operating curl on equation (15) we get,

$$
\begin{aligned}
& \rho_{\mathrm{o}} \mathrm{w}_{0} \frac{\partial}{\partial \mathrm{z}}\left(\nabla^{2} \mathrm{w}^{\prime}\right)=\rho_{0} \alpha \mathrm{g} \nabla_{1}^{2} \mathrm{~T}^{\prime}+(2 \zeta+\eta) \nabla^{4} \mathrm{w}^{\prime}+\zeta \nabla^{2}\left(\nabla \times \vec{\omega}^{\prime}\right) \\
& \left.-\frac{\Delta \mathrm{T}}{\left(1-\mathrm{e}^{\frac{\mathrm{w}_{0 \mathrm{~d}}}{\chi}}\right)} \frac{\mathrm{w}_{0}}{\chi} \mathrm{e}^{\frac{\mathrm{w}_{0 z}}{\chi}}\left[\frac{\varepsilon_{0} \mathrm{e}^{2} \mathrm{E}_{0}^{2} \nabla_{1}^{2} \mathrm{~T}^{\prime}}{\left(1+\chi_{\mathrm{e}}\right)}-\varepsilon_{0} \mathrm{eE}_{0} \nabla_{1}^{2} \mathrm{D} \varphi^{\prime}\right]\right\},
\end{aligned}
$$

$\rho_{\mathrm{o}} \operatorname{Iw}_{0}\left[\frac{\partial\left(\nabla \times \vec{\omega}^{\prime}\right)}{\partial \mathrm{z}}\right]=\eta^{\prime} \nabla^{2}\left(\nabla \times \vec{\omega}^{\prime}\right)+\zeta\left(\nabla^{2} \mathrm{w}-2 \nabla \times \vec{\omega}^{\prime}\right)$,

$\left(1+\chi_{\mathrm{e}}\right) \nabla^{2} \phi^{\prime}-\mathrm{eE}_{0} \mathrm{DT}^{\prime}=0$.

The perturbation equations (16), (20), (21) and (22) are nondimensionalised using the following definition:

$$
\left.\begin{array}{l}
\left(\mathrm{x}^{*}, \mathrm{y}^{*}, \mathrm{z}^{*}\right)=\frac{(\mathrm{x}, \mathrm{y}, \mathrm{z})}{\mathrm{d}}, \mathrm{W}^{*}=\frac{\mathrm{W}^{\prime}}{(\chi / \mathrm{d})}, \omega^{*}=\frac{\omega^{\prime}}{(\chi / \mathrm{d})}, \\
\mathrm{t}^{*}=\frac{\mathrm{t}}{\left(\mathrm{d}^{2} / \chi\right)}, \mathrm{T}^{*}=\frac{\mathrm{T}^{\prime}}{\Delta \mathrm{T}}, \phi^{*}=\frac{\phi^{\prime}}{\frac{\mathrm{eE}_{0} \Delta \mathrm{Td}}{\left(1+\chi_{\mathrm{e}}\right)}}, \Omega^{*}=\frac{\nabla \times \overrightarrow{\mathrm{w}}}{\frac{\chi}{\mathrm{d}^{3}}}
\end{array}\right\},
$$

Using equation (23) into equations (16), (20), (21) and (22) we get the dimensionless equations in the form (after neglecting the asterisks):

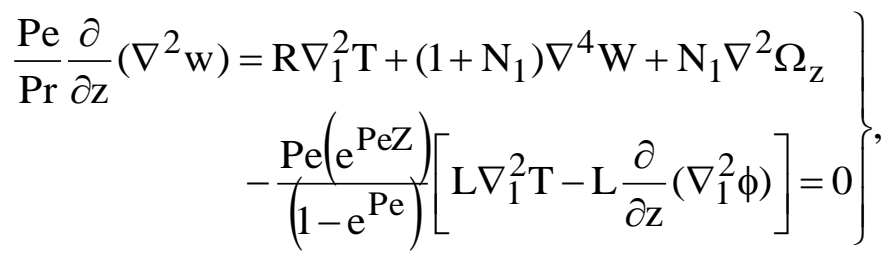

$$
\begin{aligned}
& \frac{\mathrm{N}_{2} \mathrm{P}_{\mathrm{e}}}{\operatorname{Pr}} \frac{\partial \Omega_{\mathrm{z}}}{\partial \mathrm{z}}=\mathrm{N}_{3} \nabla^{2} \Omega_{\mathrm{z}}-\mathrm{N}_{1} \nabla^{2} \mathrm{~W}-2 \mathrm{~N}_{1} \Omega_{\mathrm{z}},
\end{aligned}
$$




$$
\begin{aligned}
& \mathrm{Pe} \frac{\partial \mathrm{T}}{\partial \mathrm{z}}=\nabla^{2} \mathrm{~T}+\frac{\mathrm{Pe}\left(\mathrm{e}^{\mathrm{PeZ}}\right)}{\left(1-\mathrm{e}^{\mathrm{Pe}}\right)}\left(\mathrm{N}_{5} \Omega_{\mathrm{z}}-\mathrm{W}\right), \\
& \nabla^{2} \phi-\frac{\partial \mathrm{T}}{\partial \mathrm{z}}=0 .
\end{aligned}
$$

The non-dimensional parameters $\mathrm{N}_{1}, \mathrm{~N}_{2}, \mathrm{~N}_{3}, \mathrm{~N}_{5}, \mathrm{Pr}, \mathrm{L}, \mathrm{Pe}$ and $R$ are as defined as

$$
\begin{array}{lr}
\mathrm{N}_{1}=\frac{\zeta}{\zeta+\eta} & \text { (Coupling Parameter) } \\
\mathrm{N}_{2}=\frac{\mathrm{I}}{\mathrm{d}^{2}} & \text { (Inertia Paramter) } \\
\mathrm{N}_{3}=\frac{\eta^{\prime}}{(\zeta+\eta) \mathrm{d}^{2}} & \text { (Couple Stress Parameter) } \\
\mathrm{N}_{5}=\frac{\beta}{\rho_{\mathrm{o}} \mathrm{C}_{\mathrm{v}} \mathrm{d}^{2}} & \text { (Micropolar Heat Conduction Parameter) } \\
\mathrm{Pr}=\frac{\zeta+\eta}{\rho_{\mathrm{o}} \chi} & \text { (Prandtl Number) } \\
\mathrm{L}=\frac{\varepsilon_{0} \mathrm{e}^{2} \mathrm{E}_{0}^{2} \Delta \mathrm{T}^{2} \mathrm{~d}^{2}}{\left(1+\chi_{\mathrm{e}}\right)(\zeta+\eta) \chi} & \text { (Electric Rayleigh Number) } \\
\mathrm{Pe}=\frac{\mathrm{w}_{0} \mathrm{~d}}{\kappa} & \text { (Peclet number) } \\
\mathrm{R}=\frac{\rho_{\mathrm{o}} \alpha \mathrm{g} \Delta \mathrm{Td} \mathrm{d}^{3}}{\chi(\zeta+\eta)} & \text { (Rayleigh number) },
\end{array}
$$

The infinitesimal perturbation $\mathrm{W}, \Omega_{\mathrm{z}}, \mathrm{T}$ and $\phi$ are assumed to be periodic waves (see Chandrasekhar [36]) and hence these permit a normal mode solution in the form 
$\left[\begin{array}{l}\mathrm{W} \\ \Omega_{\mathrm{z}} \\ \mathrm{T} \\ \phi\end{array}\right]=\left[\begin{array}{c}\mathrm{W}(\mathrm{z}) \\ \mathrm{G}(\mathrm{z}) \\ \mathrm{T}(\mathrm{z}) \\ \phi(\mathrm{z})\end{array}\right] \mathrm{e}^{\mathrm{i}(\mathrm{lx}+\mathrm{my})}$

where 1 and $\mathrm{m}$ are horizontal components of the wave number $\mathrm{a}^{2}$, Substituting equation (28) into equations (24)-(27), we get

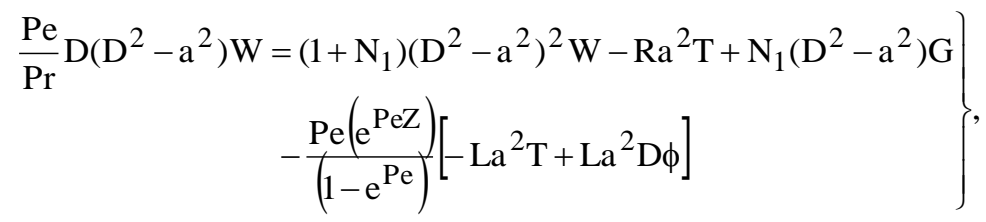

$\frac{N_{2} P e}{\operatorname{Pr}} D G=N_{3}\left(D^{2}-a^{2}\right) G-N_{1}\left(D^{2}-a^{2}\right) W-2 N_{1} G$,

$\mathrm{PeDT}=\left(\mathrm{D}^{2}-\mathrm{a}^{2}\right) \mathrm{T}+\frac{\mathrm{Pe}\left(\mathrm{e}^{\mathrm{PeZ}}\right)}{\left(1-\mathrm{e}^{\mathrm{Pe}}\right)}\left(\mathrm{W}-\mathrm{N}_{5} \mathrm{G}\right)$,

$\left(\mathrm{D}^{2}-\mathrm{a}^{2}\right) \phi-\mathrm{DT}=0$

where, $D=\frac{d}{d z}$.

Equations (26)-(29) are solved using the Galerkin technique. Multiplying equation (29) by $\mathrm{W}$, equation (30) by $\mathrm{G}$, equation (31) by $T$, and equation (32) by $\phi$ and integrating the resulting equation by parts with respect to $\mathrm{z}$ from 0 to 1 and taking $\mathrm{W}=\mathrm{AW}, \mathrm{G}=\mathrm{BG}_{1}$ , $\mathrm{T}=\mathrm{CT}_{1}$ and $\phi=\mathrm{E} \phi_{1}$ in which $\mathrm{A}, \mathrm{B}, \mathrm{C}$ and $\mathrm{E}$ are constants with $\mathrm{W}_{1}, \mathrm{G}_{1}, \mathrm{~T}_{1}$ and $\phi_{1}$ are trial functions. This procedure yields the following equation for the Rayleigh number $\mathrm{R}$ :

$$
\mathrm{R}=\frac{\mathrm{Y}_{1} \mathrm{Y}_{3} \mathrm{Y}_{4}}{\mathrm{a}^{2}\left\langle\mathrm{~W}_{1} \mathrm{~T}_{1}\right\rangle \mathrm{Y}_{2}}+\mathrm{L} \frac{\mathrm{Y}_{5}}{\left\langle\mathrm{~W}_{1} \mathrm{~T}_{1}\right\rangle\left\langle\phi_{1}\left(\mathrm{D}^{2}-\mathrm{a}^{2}\right) \phi_{1}\right\rangle},
$$

where, 


$$
\begin{aligned}
\mathrm{Y}_{1} & =\mathrm{N}_{3}\left\langle\mathrm{G}_{1}\left(\mathrm{D}^{2}-\mathrm{a}^{2}\right) \mathrm{G}_{1}\right\rangle-2 \mathrm{~N}_{1}\left\langle\mathrm{G}_{1}^{2}\right\rangle, \\
\mathrm{Y}_{2} & =\mathrm{N}_{1} \mathrm{~N}_{5}\left\langle\mathrm{G}_{1}\left(\mathrm{D}^{2}-\mathrm{a}^{2}\right) \mathrm{W}_{1}\right\rangle\left\langle\mathrm{T}_{1} \mathrm{G}_{1} \mathrm{f}(\mathrm{z})\right\rangle-\left\langle\mathrm{T}_{1} \mathrm{~W}_{1} \mathrm{f}(\mathrm{z})\right\rangle \mathrm{Y}_{1}, \\
\mathrm{Y}_{3} & =\mathrm{P}_{\mathrm{e}}\left\langle\mathrm{T}_{1} \mathrm{DT} \mathrm{T}_{1}\right\rangle-\left\langle\mathrm{T}_{1}\left(\mathrm{D}^{2}-\mathrm{a}^{2}\right) \mathrm{T}_{1}\right\rangle, \\
\mathrm{Y}_{4} & \left.=\left(1+\mathrm{N}_{1}\right)\left\langle\mathrm{W}_{1}\left(\mathrm{D}^{2}-\mathrm{a}^{2}\right)^{2} \mathrm{~W}_{1}\right\rangle-\frac{\mathrm{P}_{\mathrm{e}}}{\mathrm{Pr}}\left\langle\mathrm{W}_{1} \mathrm{D}\left(\mathrm{D}^{2}-\mathrm{a}^{2}\right) \mathrm{W}_{1}\right\rangle\right) \\
& +\mathrm{N}_{1}^{2} \frac{\left\langle\mathrm{G}_{1}\left(\mathrm{D}^{2}-\mathrm{a}^{2}\right) \mathrm{W}_{1}\right\rangle\left\langle\mathrm{W}_{1}\left(\mathrm{D}^{2}-\mathrm{a}^{2}\right) \mathrm{G}_{1}\right\rangle}{\mathrm{Y}_{1}} \\
\mathrm{Y}_{5} & =\left\langle\mathrm{W}_{1} \mathrm{~T}_{1} \mathrm{f}(\mathrm{z})\right\rangle\left\langle\phi_{1}\left(\mathrm{D}^{2}-\mathrm{a}^{2}\right) \phi_{1}\right\rangle-\left\langle\mathrm{W}_{1} \mathrm{D} \phi_{1} \mathrm{f}(\mathrm{z})\right\rangle\left\langle\phi_{1} \mathrm{DT} \mathrm{T}_{1}\right\rangle .
\end{aligned}
$$

In the equation (33), $\langle---\rangle$ denotes integration with respect to $\mathrm{Z}$ between $\mathrm{z}=0$ and $\mathrm{z}=1$. We note here that $\mathrm{R}$ in equation (33) is a functional and the Euler-Lagrange equations for the extremisation of $\mathrm{R}$ are equations (29)-(32).

The value of critical Rayleigh number depends on the boundaries. In this paper we consider the following boundary combinations:

Free-free isothermal and no spin.

$$
\mathrm{W}=\mathrm{D}^{2} \mathrm{~W}=\mathrm{T}=\mathrm{G}=\mathrm{D} \phi=0, \quad \text { at } \quad \mathrm{z}=0,1 .
$$

Rigid-rigid isothermal and no spin.

$$
\mathrm{W}=\mathrm{DW}=\mathrm{T}=\mathrm{G}=\mathrm{D} \phi=0, \quad \text { at } \quad \mathrm{z}=0,1 .
$$

Rigid-free isothermal and no spin.

$$
\begin{aligned}
& \mathrm{W}=\mathrm{DW}=\mathrm{T}=\mathrm{G}=\mathrm{D} \phi=0, \quad \text { at } \quad \mathrm{z}=0 \text {, } \\
& \mathrm{W}=\mathrm{D}^{2} \mathrm{~W}=\mathrm{T}=\mathrm{G}=\mathrm{D} \phi=0, \quad \text { at } \quad \mathrm{z}=1 \text {. }
\end{aligned}
$$

Free-free adiabatic and no spin.

$$
\mathrm{W}=\mathrm{D}^{2} \mathrm{~W}=\mathrm{DT}=\mathrm{G}=\mathrm{D} \phi=0, \quad \text { at } \quad \mathrm{z}=0,1 .
$$

(a) Rigid-rigid isothermal and no spin.

$$
\mathrm{W}=\mathrm{DW}=\mathrm{DT}=\mathrm{G}=\mathrm{D} \phi=0, \quad \text { at } \mathrm{z}=0,1 .
$$


(b) Rigid-free isothermal and no spin.

$$
\begin{aligned}
& \mathrm{W}=\mathrm{DW}=\mathrm{DT}=\mathrm{G}=\mathrm{D} \phi=0, \quad \text { at } \quad \mathrm{z}=0, \\
& \mathrm{~W}=\mathrm{D}^{2} \mathrm{~W}=\mathrm{DT}=\mathrm{G}=\mathrm{D} \phi=0, \quad \text { at } \quad \mathrm{z}=1 .
\end{aligned}
$$

The trial functions satisfying the boundary conditions are presents in Table 1.

Table 1: Trial functions for the different for the different boundary conditions.

\begin{tabular}{|l|c|c|c|}
\hline $\begin{array}{l}\text { Boundary } \\
\text { conditions }\end{array}$ & Free-free & Rigid-rigid & Rigid-free \\
\hline $\mathrm{W}_{1}$ & $\mathrm{z}^{4}-2 \mathrm{z}^{3}+\mathrm{z}$ & $\mathrm{W}_{1}=\mathrm{z}^{4}-\frac{5}{2} \mathrm{z}^{3}+\frac{3}{2} \mathrm{z}^{2}$ & $\mathrm{~W}_{1}=\mathrm{z}^{4}-2 \mathrm{z}^{3}+\mathrm{z}^{2}$ \\
\hline $\mathrm{G}_{1}$ & $\mathrm{z}(\mathrm{z}-1)$ & $\mathrm{z}(\mathrm{z}-1)$ & $\mathrm{z}(\mathrm{z}-1)$ \\
\hline$\phi_{1}$ & $\mathrm{z}^{2}(3-2 \mathrm{z})$ & $\mathrm{z}^{2}(3-2 \mathrm{z})$ & $\mathrm{z}^{2}(3-2 \mathrm{z})$ \\
\hline $\begin{array}{l}\text { Isothermal } \\
\mathrm{T}_{1}\end{array}$ & $\mathrm{z}(1-\mathrm{z})$ & $\mathrm{z}(1-\mathrm{z})$ & $\mathrm{z}(1-\mathrm{z})$ \\
\hline $\begin{array}{l}\text { Adiabatic } \\
\mathrm{T}_{1}\end{array}$ & 1 & 1 & 1 \\
\hline
\end{tabular}

\section{Results and Discussion}

In this chapter, we study the effects of suction-injectioncombination and electric field on the onset of Rayleigh-Bénard convection in a micropolar fluid.

Before discussing the results obtained in this paper, the following observations are made from the equation (33). In the case of symmetric boundaries (free-free and rigid - rigid) the value of $R_{c}$ is same for both pro and anti-gravity suction-injection-combinations as $R_{c}$ is an even function of Pe and in the case of non-symmetric boundary combination (rigid - free) $R_{c}$ is not an even function, thus the value of $R_{c}$ is not the same for both $\mathrm{Pe}<0$ and $\mathrm{Pe}>0$ even in the absence of magnetic field.

The critical Rayleigh number $R_{c}$ obtained using Galerkin method for different values of $\mathrm{N}_{1}, \mathrm{~N}_{3}, \mathrm{~N}_{5}, \mathrm{~L}$, and $\operatorname{Pr}$ are shown in figures (3) - (14). Figures (3) - (8) pertain to different velocity boundary 
combination and isothermal temperature boundaries and figures (9) - (14) pertain to adiabatic temperature boundaries.

Figure (3) is the plot of $R_{c}$ versus Pe for different values of coupling parameter, $\mathrm{N}_{1}$, and for different velocity boundary combinations with isothermal temperature conditions. It is found that $R_{c}$ increases with the increase in $\mathrm{N}_{1} . \mathrm{N}_{1}$ represents the concentration of microelements in the fluid. When N1 increases concentration of the microelements are also increases, this microelelmetns consume greater part of the energy in forming the gyrational velocity, which delays the onset of convection. From this we conclude that increase in concentration of microelements stabilizes the system.

Figure (4) are the plot of $R_{c}$ versus Pe for different values of couple stress parameter, $\mathrm{N}_{3}$, for different velocity boundary combinations with isothermal temperature conditions. From the figure we observe that the increase in $\mathrm{N}_{3}$ decreases $\mathrm{R}_{\mathrm{c}}$. This is because, when $\mathrm{N}_{3}$ increases the couple stress of the fluid increases, which decreases the microrotation and thereby destabilizing the system.

Figure (5) are the plot of $R_{c}$ versus Pe for different values of micropolar heat conduction parameter, $\mathrm{N}_{5}$, for different velocity boundary combinations with isothermal temperature conditions. Clearly, $R_{c}$ increase with $N_{5}$. When $\mathrm{N}_{5}$ increases, the heat induced into the fluid due to microelements also increases, thus reducing the heat transfer from bottom to top. The decrease in heat transfer is responsible for delaying the onset of instability. Thus, the effect of $\mathrm{N}_{5}$ is to stabilizes the system.

Figure (6) are the plot of Rc versus Pe for different values of electric Rayleigh number, L, for different velocity boundary combinations with isothermal temperature conditions. It is found that as $\mathrm{L}$ increases, $R_{c}$ decreases. Thus, $L$ destabilizes the system.

Figures (7) are the plot of $R_{c}$ versus Pe for different values of Prandtl number, Pr, for different velocity boundary combinations with isothermal temperature conditions. From the figure it observed that in the case of anti-gravity SIC increase in Pr, increases $R_{c}$, thus stabilizing the system. In the case of pro-gravity SIC we see that $R_{c}$ decreases with an increase in $\operatorname{Pr}$ and thereby destabilizes the system. Thus in deciding stability of the system Prandtl number plays an important role. 
Figures (8) - (12) are plots for adiabatic boundaries corresponding to figures (3) - (7) of isothermal boundaries. The results of adiabatic boundaries are qualitatively similar to that of isothermal boundaries.

From the above figures the following points are noted:

1) In the case of anti-gravity SIC, i.e., $\mathrm{Pe}>0$, increase in Pe decreases $R_{c}$ initially (for small values of Pe up to a certain critical value of Pe namely $\mathrm{Pe}_{c}$ ) and further increase in $\mathrm{Pe}$ beyond $\mathrm{Pe}_{\mathrm{c}}$ increases the $\mathrm{R}_{\mathrm{c}}$. Thus the weak anti-gravity SIC in micropolar fluids destabilizes the system. This may be due to distortion of the basic temperature profile caused by SIC. Physically this amounts to an increase in the rate at which energy is supplied to the disturbance. In the case of pro-gravity SIC, i.e., $\mathrm{Pe}<0$, increase in Pe increases $\mathrm{R}_{c}$ and thus the pro-gravity SIC makes the system stable.

2) We also find that $R_{c}^{R R}>R_{c}^{R F}>R_{c}^{F F}$, where the superscripts correspond to the three different velocity boundary combinations. Thus rigid-rigid boundary is more stable compared to other velocity boundary combinations.

3) In the case of adiabatic boundary the convection sets in at wave number, $\mathrm{a}_{\mathrm{c}}=0$.

4) 4) It is also observed from the table that $\mathrm{Pe}_{\mathrm{c}}^{\mathrm{RR}}<\mathrm{Pe}_{\mathrm{c}}^{\mathrm{FF}}<\mathrm{Pe}_{\mathrm{c}}^{\mathrm{RF}}$. The behavior of $\mathrm{Pe}_{\mathrm{c}}$ in adiabatic case is quantitatively similar to that observed in the case of isothermal boundaries.

\section{Conclusion}

It is found that the effect of SIC is to stabilize or destabilize accordingly SIC are Pro-gravity and anti-gravity. Thus, by adjusting the suction-injection-combination and Prandtl number, $\mathrm{Pr}$ it is possible to control the convection in a micropolar fluid with electric field. 


\section{References}

[1] H. Power, Bio-Fluid Mechanics, Advances in Fluid Mechanics, W.I.T. Press, U.K. 1995.

[2] G. Lukaszewicz, Micropolar fluid theory and applications, Birkhauser, Boston. 1999.

[3] A. C. Eringen, Micro Continuum field theory, Springer Verlag, New York. 1999.

[4] A. C. Eringen, "Theory of micropolar fluids, Journal of Mathematics and Mechanics," vol. 16, pp. 1-18, 1996.

[5] A. B. Datta, and V. U. K. Sastry, "Thermal instability of a horizontal layer of micropolar fluid heated from below," International Journal of Engineering Science, vol. 14, no. 7, pp. 631-637, 1976.

[6] G. Ahmadi, "Stability of a micropolar fluid layer heated from below," International Journal of Engineering Science, vol. 14, no. 1, pp. 81-89, 1967.

[7] S. P. Bhattacharyya, and S. K. Jena, "On the Stability of Hot Layer of Micropolar Fluid," International Journal of Engineering Science, vol. 21, no. 9, pp. 1019-1024, 1983.

[8] P. G. Siddheshwar, and S. Pranesh, "Magnetoconvection in a micropolar fluid," International Journal of Engineering Science, USA, vol. 36, pp. 1173-1181, 1998.

[9] P. G. Siddheshwar, and S. Pranesh, "Effects of non-uniform temperature gradients and magnetic field on the onset of convection in fluids with suspended particles under microgravity conditions," Indian Journal of Engineering and Materials Sciences, vol. 8, pp. 77-83, 2001.

[10] P. G. Siddheshwar, and S. Pranesh, "Effect of temperature / gravity modulation on the onset of magneto-convection in weak electrically conducting fluids with internal angular momentum," Journal of Magnetism and Magnetic Materials, USA, vol. 192, pp. 159-176, 1999.

[11] P. G. Siddheshwar, and S. Pranesh, "Magnetoconvection in fluids with suspended particles under $1 \mathrm{~g}$ and $\mu \mathrm{g}$," 
International Journal of Aerospace Science and Technology, France, vol. 6, pp. 105-114, 2001.

[12] S. Pranesh, and Riya Baby, "Effect of Non-Uniform Temperature Gradient on the Onset of Rayleigh-Bénard Electro Convection in a Micropolar Fluid," Applied Mathematics, vol. 3, pp. 442-450, 2012.

[13] S. Pranesh and N. Arun Kumar, "Effect of Non-Uniform Basic Concentration Gradient on the Onset of DoubleDiffusive Convection in Micropolar Fluid". Applied Mathematics, vol. 3, pp. 417-424, 2012.

[14] H. Bénard, "Les tourbillions cellularies dans une nappe liquide transportant de la chaleut par convection en regime permanent, Annales de chimie et de physique, vol. 23, pp. 62. 1901.

[15] D. L. Shvartsblat, "Steady convection motions in a plane horizontal fluid layer with permeable boundaries," Fluid Dynamics, vol. 4, No. 5, pp. 54-59, 1969.

[16] D. A. Nield, "Through flow effects in the Rayleigh-Bénard convective instability problem," Journal of Fluid Mechanics, vol. 185, pp.353-360, 1987.

[17] P. G. Siddheshwar, and S. Pranesh, "Suction-Injection effects on the onset of Rayleigh-Bénard-Marangoni convection in a fluid with suspended particles," Acta Mechanica, vol. 152, pp. 241-252, 2001.

[18] I. S. Shivakumara, and S. P. Suma, "Effects of through flow and internal heat generation on the onset of convection in a fluid layer," Acta Mechanica, vol. 140, No. 3, pp. 207-217, 2000.

[19] Y. N. Murty, and V. V. Ramana Rao, "Effect of through flow on Marangoni convection in micropolar fluids," Acta Mechanica, vol. 138, No. 3, pp. 211, 2000.

[20] Y. N. Murty, "Effect of throughflow and magnetic field on Bénard convection in micropolar fluids". Acta Mechanica, vol. 150, No. 1, pp. 11-21, 2001. 
[21] Y. N. Murty, "Effect of throughflow and magnetic field on Marangoni convection in micropolar fluids," Applied Mathematics and Computation, vol. 173, pp. 1288-1299. 2006.

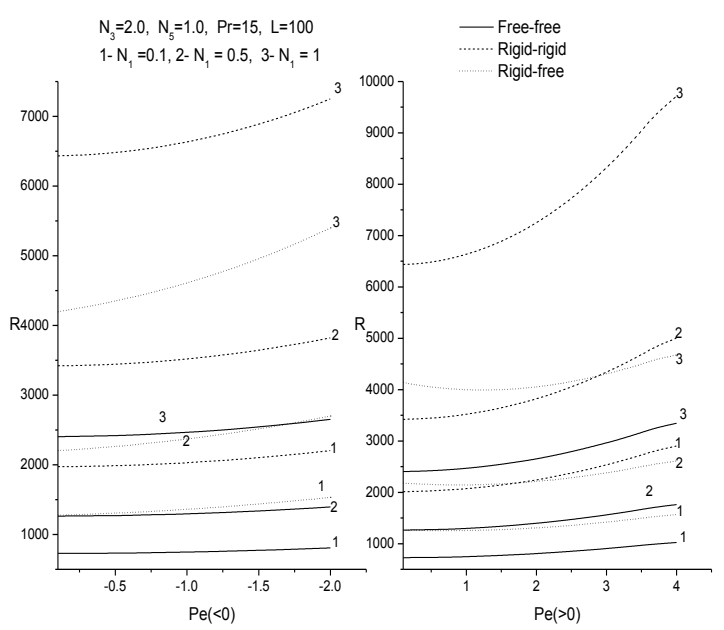

Fig 2: Plot of Rayleigh number R versus Peclet number Pe for different values of coupling parameter $\mathrm{N}_{1}$ for different velocity boundary combinations and isothermal temperature condition.

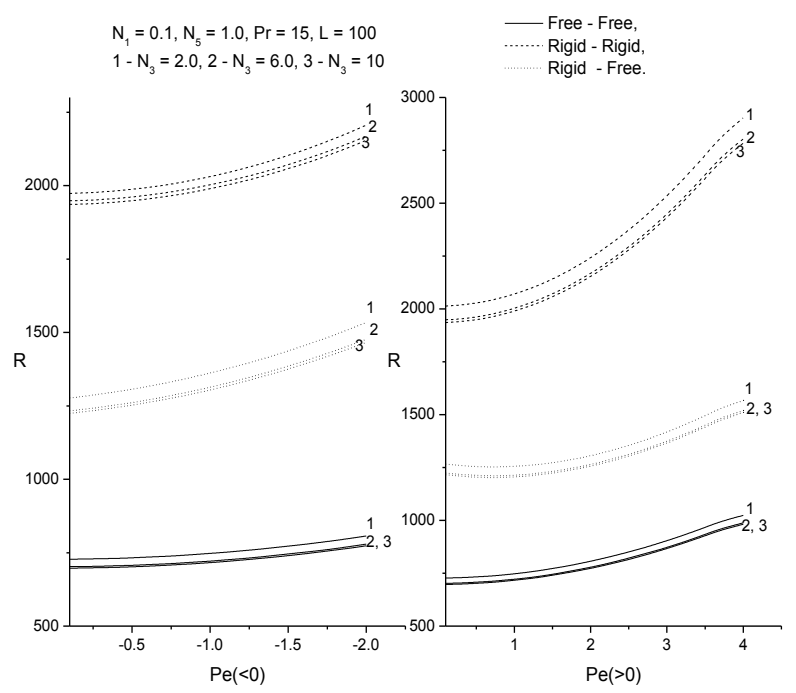

Fig 3: Plot of $\mathrm{R}$ versus Pe for different values of couple stress parameter $\mathrm{N}_{3}$ for different velocity boundary combinations and isothermal temperature condition. 


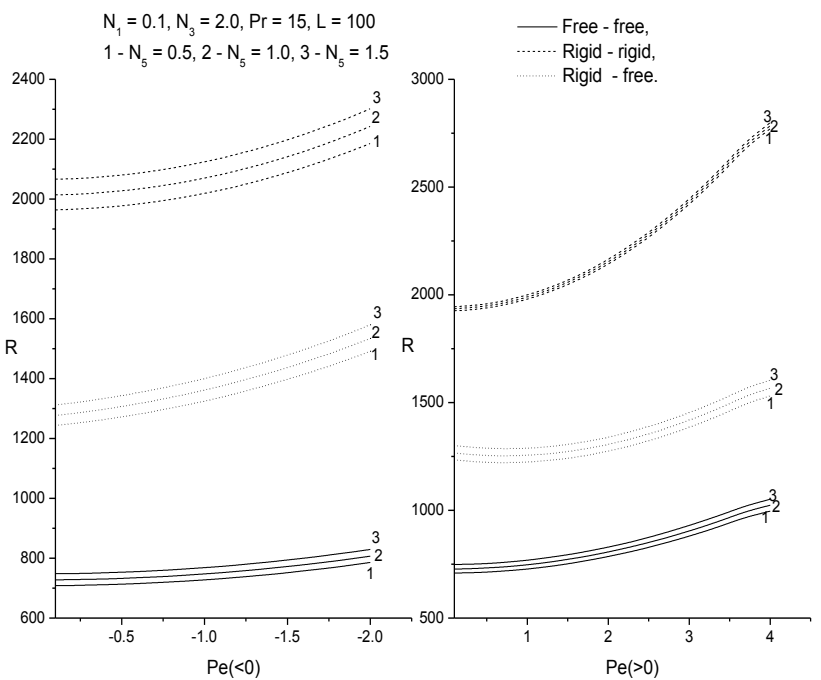

Fig 4: Plot of $\mathrm{R}$ versus Pe for different values of micropolar heat conduction parameter $\mathrm{N}_{5}$ for different velocity boundary combinations and isothermal temperature condition.

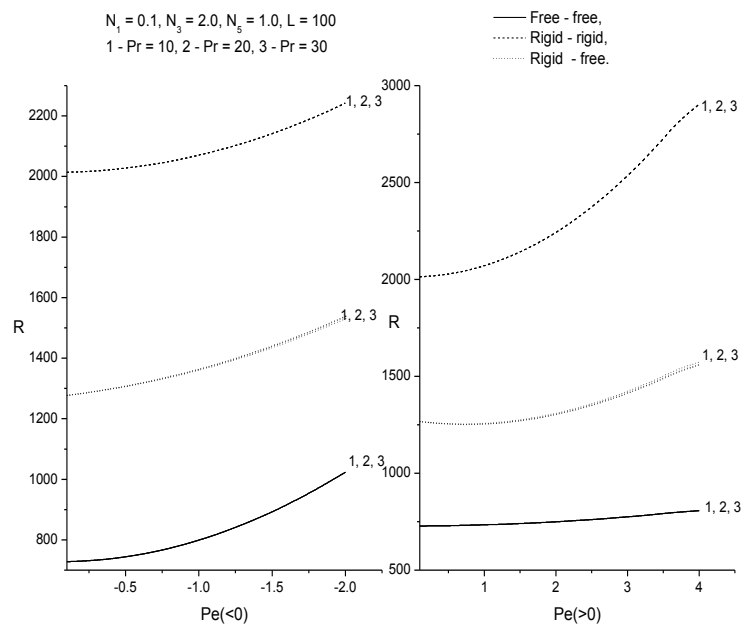

Fig 5: Plot of R versus Pe for different values of Prandtl number Pr for different velocity boundary combinations and isothermal temperature condition. 


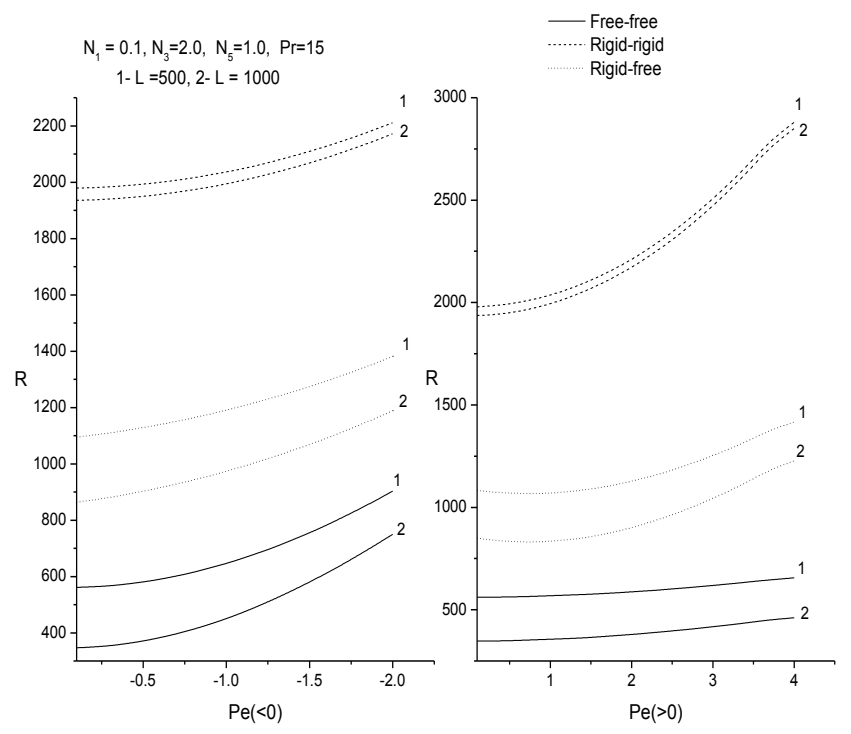

Fig 6: Plot of R versus Pe for different values of Electric Rayleigh number L for different velocity boundary combinations and isothermal temperature condition.

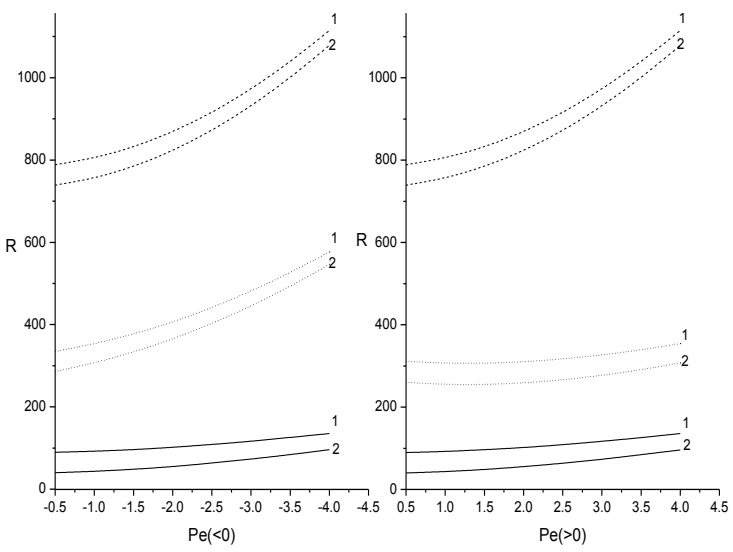

Fig 7: Plot of R versus Pe for different values of Electric Rayleigh number L for different velocity boundary combinations and adiabatic temperature condition. 


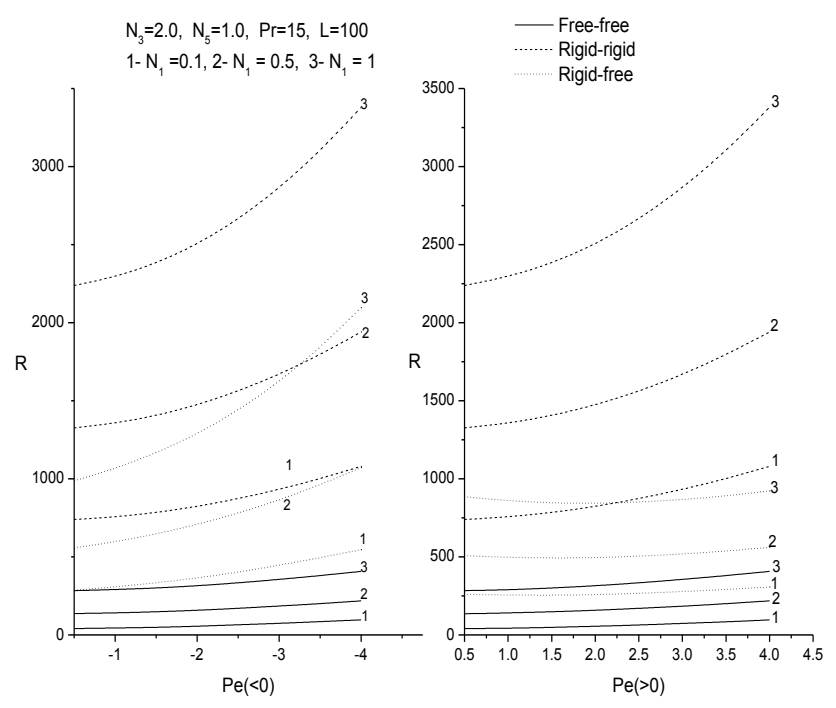

Fig 8: Plot of $\mathrm{R}$ versus Pe for different values of coupling parameter $\mathrm{N}_{1}$ for different velocity boundary combinations and adiabatic temperature condition.

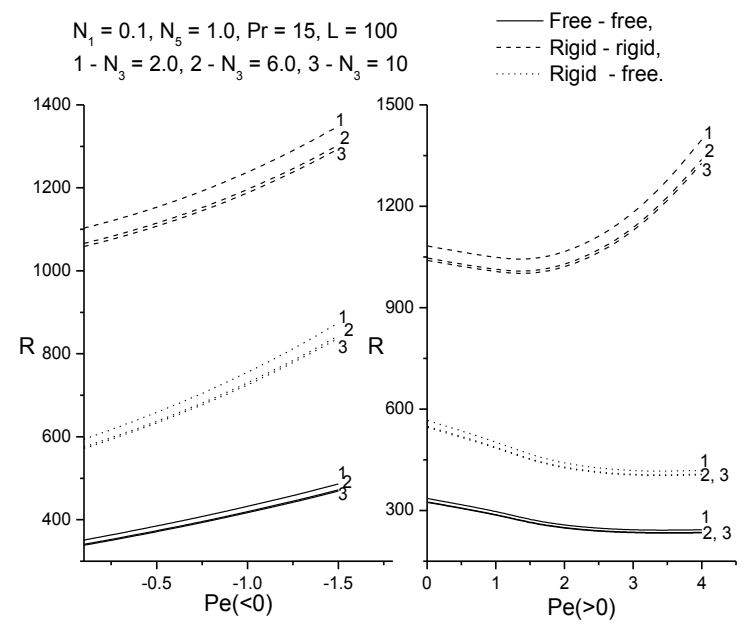

Fig 9: Plot of $\mathrm{R}$ versus Pe for different values of couple stress parameter $\mathrm{N}_{3}$ for different velocity boundary combinations and adiabatic temperature condition. 


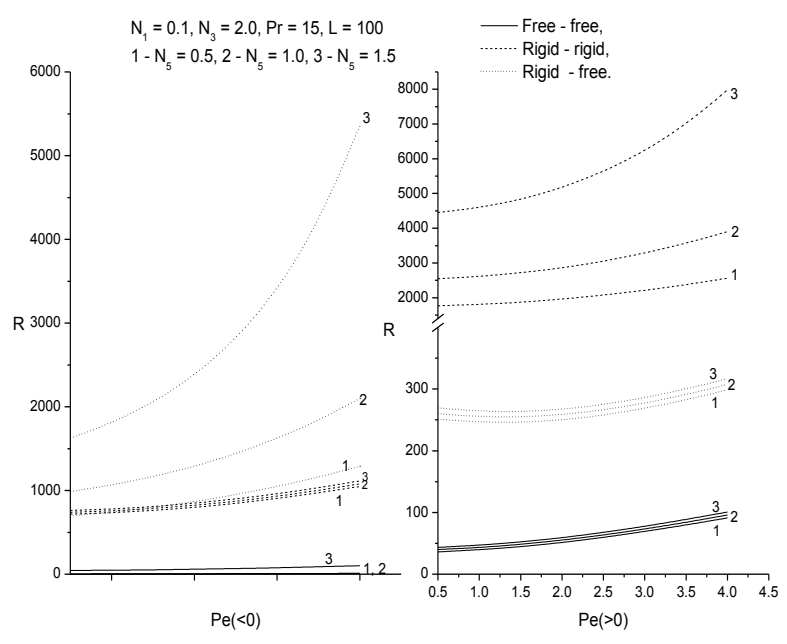

Fig 10: Plot of $\mathrm{R}$ versus $\mathrm{Pe}$ for different values of micropolar heat conduction parameter $\mathrm{N}_{5}$ for different velocity boundary combinations and adiabatic temperature condition.

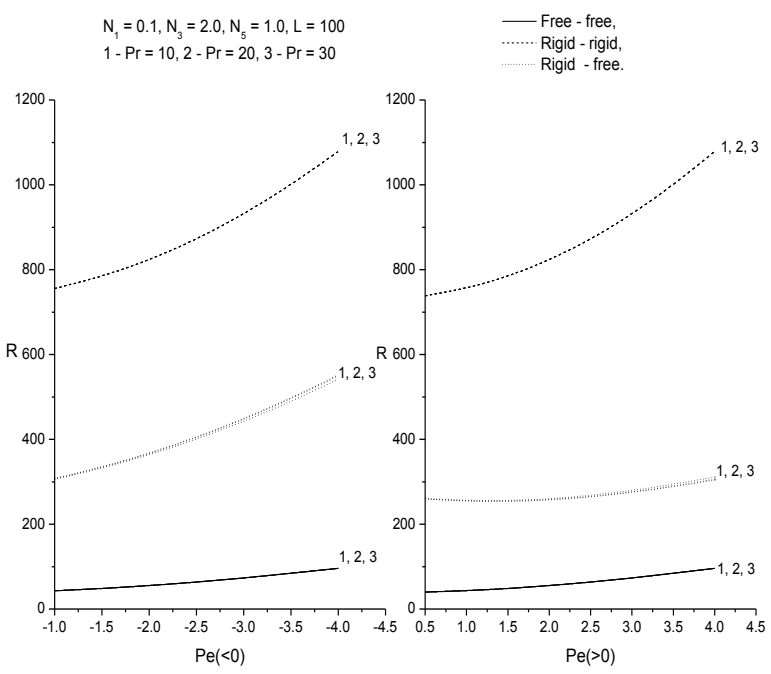

Fig 11: Plot of R versus Pe for different values of Prandtl number Pr for different velocity boundary combinations and adiabatic temperature condition. 